\title{
Large-Scale Integration of Nanomechanical Sensors
}

\author{
S. Evoy \\ Department of Electrical and Computer Engineering, University of Alberta, and National Institute for \\ Nanotechnology, 9107 - 116th St., Edmonton, Alberta, T6G 2V4, Canada
}

\begin{abstract}
Rapid analysis of biological molecules is of great importance to many biomedical applications. Mechanical resonators have been demonstrated as highly sensitive transducers for the detection of molecular systems. Akin to the concept of chemoresistive electronic noses, large arrays of nanomechanical resonators would enable the development of nanoresonator-based 'electronic ears' for the fingerprinting of metabolomics systems. We will present a review of our work involving the large scale integration of nanoresonating sensors, and their application for the specific detection of biological targets.
\end{abstract}

Key words: NEMS, Nanoresonators, Large-Scale Integration, Electronic Noses.

\section{Introduction}

Detection of biological and chemical agents is critical to many life sciences areas including disease diagnosis, drug molecule screening, and rapid analysis of various molecular systems. Micro- and nanoresonators have also been shown to be promising platforms for such tagless analysis. The binding of the analyte onto the sensor surface is detected through a shift of resonant frequency induced by its added mass. The mass sensitivity of mechanical resonators scales favorably as their mass is reduced, offering a compelling path for the development of sensors of exceptional sensitivities. We have recently reported a novel SiCN material of tunable properties for the highyield fabrication of NEMS [1]. The residual stress of this material can be tuned through post-residual anneal, allowing full access from the compressive to the tensile range. These properties as well as optimizations in the fabrication procedure have allowed the machining of resonators as narrow as $\sim 25 \mathrm{~nm}$ with a yield approaching $100 \%$. In addition, this approach also does not require a critical point drying step which is known to leave residues onto the device surfaces. The resonant response of the devices is measured by optical interferometry [2].

Nanoimprint lithography was also used to fabricate arrays of SiCN nanoscale resonators for biological analysis applications. A bilayer resist consisting of PMMA 495/LOR 3A allowed high fabrication yields for resonators of widths ranging from $120 \mathrm{~nm}$ to $300 \mathrm{~nm}$, thicknesses of $40 \mathrm{~nm}$ and $70 \mathrm{~nm}$, and a length of $14 \mu \mathrm{m}$. To our knowledge, these $120 \mathrm{~nm}$ resonators are the narrowest suspended structures ever fabricated via nanoimprinting.

The last few years have also seen the development of alternate "bottom-up" techniques for the fabrication of nanodevices. For example, silicon nanowires have been used to detect biological and chemical species [3]. These sensors operate by monitoring changes of electrical conductivity associated to the binding. In this work, the specific detection of proteins using nanowires was achieved through the monitoring of their mechanical resonance. The specific detection of streptavidin and protein A are demonstrated as proof of concept.

\section{Experimental}

$50 \mathrm{~nm}$ thick SiCN layer was deposited by plasma-enhanced chemical vapor deposition (PECVD) onto single-crystal (100) silicon wafers (500 um-thick, $100 \mathrm{~mm}$-diameter). The SiCN-coated wafers were then annealed in a tube furnace at $500 \mathrm{C}$ for 6 hours which resulted in a tensile stress of $\sim 200 \mathrm{MPa}$. Next, the resonator beams and the supporting pads were patterned using electron beam lithography A $30 \mathrm{~nm}$ thick $\mathrm{Cr}$ film, deposited by thermal evaporation and subsequently lifted off in acetone, was used as a mask for reactive ion etching. Finally, the resonators were released by anisotropic etch in $\mathrm{KOH}$ solution (35\%) saturated with IPA.

This SiCN technology was subsequently employed in conjunction with nanoimprint lithography using a bilayer of PMMA 495/LOR as imprint resist. Two bilayer thicknesses were 
investigated. A $150 \mathrm{~nm} / 150 \mathrm{~nm}$ bilayer was initially employed, and reproducibly yielded suspended beams as narrow as $300 \mathrm{~nm}$. A 100 $\mathrm{nm} / 100 \mathrm{~nm}$ bilayer was then employed, which yielded released devices as narrow as $120 \mathrm{~nm}$. The imprint itself was performed using a Nanonex NX-2500 system at a temperature of $190^{\circ} \mathrm{C}$, a pressure of $200 \mathrm{psi}$, and hold time of 2 min. An oxygen plasma was then employed to remove any resist residue at the bottom of the pattern. This oxygen plasma cleaning was performed in a Trion RIE, and at low pressures to ensure etch anisotropy $(10 \mathrm{mT}, 7 \mathrm{sccm} \mathrm{O2}$, $60 \mathrm{~W})$. The etch time employed varied from 60 $\mathrm{s}$ to $120 \mathrm{~s}$ given that the residual layer height was slightly different for each imprint.

Bottom-up silicon nanoresonators were grown onto a silicon-on-insulator (SOI) wafer that consisted of a $7 \mu \mathrm{m}$ thick (110) device layer, and a $100 \mathrm{~nm}$ thick buried oxide layer [4]. Vertical $\{111\}$ sidewalls were formed by patterning trenches in the (110) top silicon device layer using $\mathrm{KOH}$. Gold catalyst particles were then deposited from colloidal suspension using a drop-and-dry process. The CVD growth was performed using silane, $\mathrm{HCl}$, and $\mathrm{B} 2 \mathrm{H} 6$ as precursors. Typical nanowires are shown in Fig. $1 \mathrm{~b}$. Their lengths and diameters ranged from 3 to $4 \mu \mathrm{m}$ and from 20 to $150 \mathrm{~nm}$, respectively.

The chips were mounted onto a piezoelectric element which was actuated by the tracking output of a spectrum analyzer (Agilent model 4411B). The beam of a laser diode $(\lambda=655$ $\mathrm{nm}$ ) was directed through a beamsplitter and focused onto the substrate using a $N A=0.45$ microscope objective. At resonance, motion of the nanoresonators relative to the substrate created a moving fringe pattern that was reflected back through the microscope objective, was redirected by the beamsplitter, and impinged on an AC coupled photodetector (New Focus model 1601).

A vapor-deposited monolayer of 3mercaptopropyl trimethoxysilane was used to attach linkers onto these devices. Their resonance of the nanowires was then assessed immediately prior and after exposure to the target protein.

\section{Results and Discussion}

Figure 1 shows typical device arrays produced using the top down SiCN. Resonators as narrow as $8 \mathrm{~nm}$ have been obtained using electron beam lithography exposure [5-6]

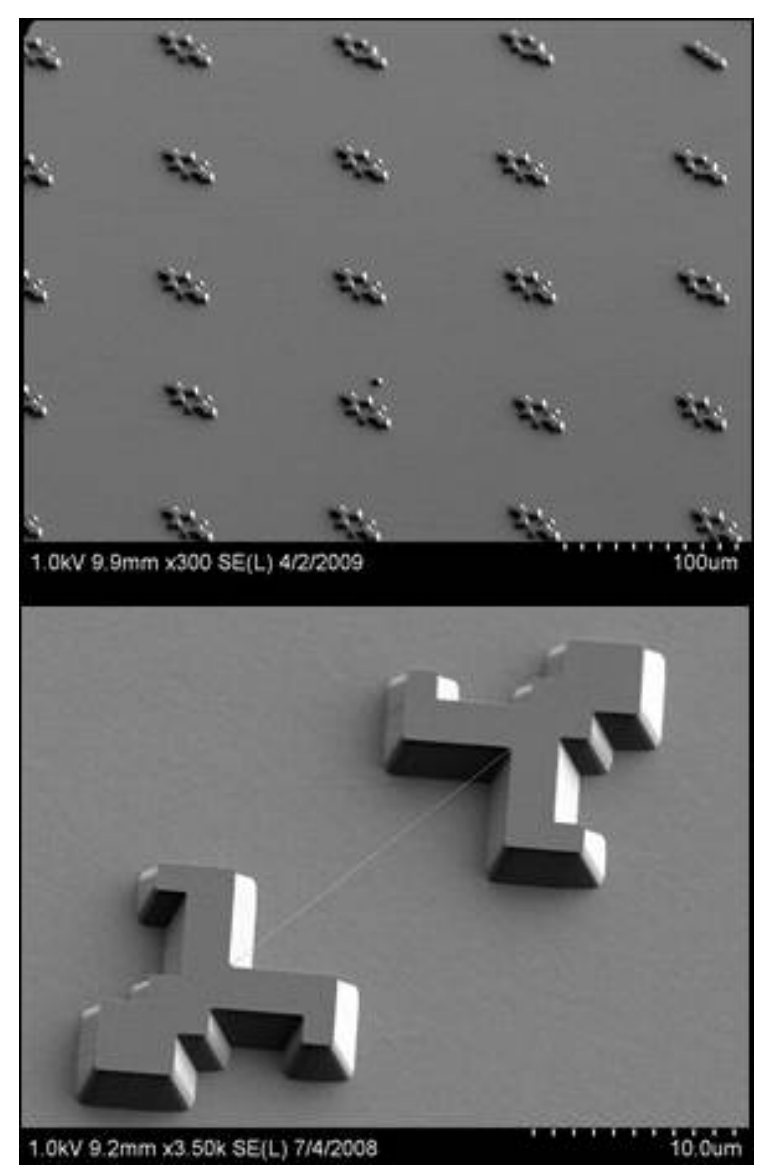

Fig. 1. Top: SEM image of a $5 \times 5$ array of doublyclamped $25 \mathrm{~nm}$ wide nanomechanical resonators with a yield of $100 \%$. Bottom: Close-up image of a 15 $\mathrm{nm}$ wide and 24 um long resonator

Immobilization of antibody fragments onto arrays of $25 \mathrm{~nm}$-wide devices resulted in consistent resonant frequency downshifts of $341 \mathrm{kHz}$. The frequencies further downshift by $216 \mathrm{kHz}$ due to protein A attachment (Fig. 2). Another "negative control" array II (Fig. 3) was processed identically except protein-A was not added to the solution. The frequency shifts due to this "solvent only" step are much smaller ( $54 \mathrm{kHz}$ ), further supporting that the significant frequency downshifts in array I are indeed due to protein-A capture. 


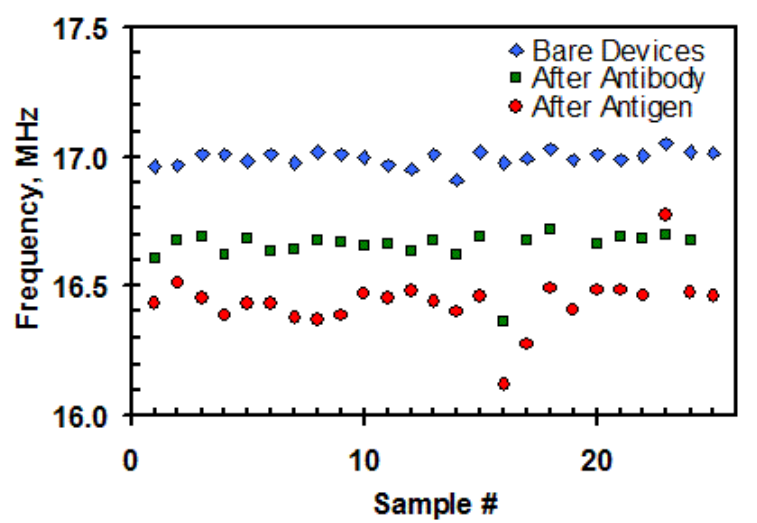

Fig. 2. Resonant frequencies of the 25 individual resonators of Array I. The blue diamonds, green squares and red circles designate the individual device frequencies of the bare reso-nators, resonators with immobilized antibodies and resonators with captured protein A, respectively. Immobiliza-tion of antibodies results in frequency downshifts of $\sim 341$ $\mathrm{kHz}$. Capture of protein A further down-shifts the frequencies by $\sim 216 \mathrm{kHz}$

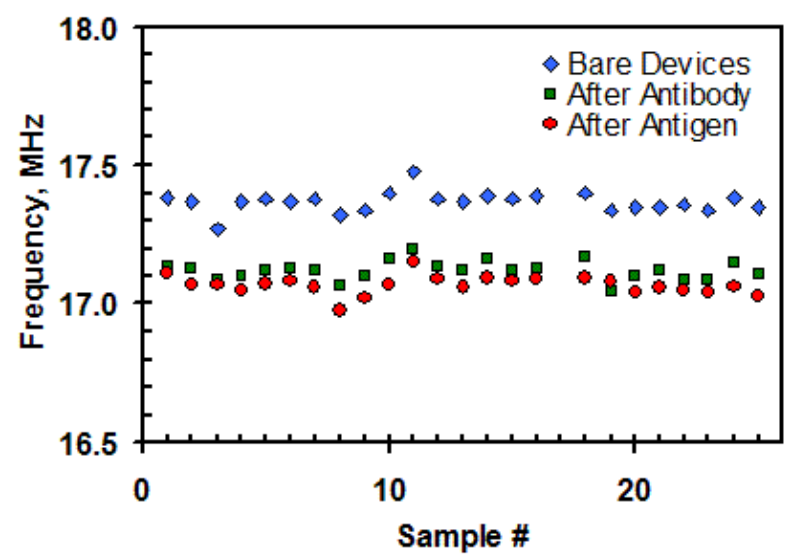

Fig. 3. Resonant frequencies of the 25 individual resonators of Array II. This "negative control" array was processed identically except protein $A$ was not added to the solution in the detection step. Similarly to Array I, immobilization of antibodies results in frequency downshifts of $\sim 248 \mathrm{kHz}$. The second "solvent only" step results in much smaller frequency downshifts of $\sim 54 \mathrm{kHz}$.

Figure 4 shows a typical $300 \mathrm{~nm}$-wide resonator structure realized via the imprinting of a $300 \mathrm{~nm}$ thick PMMA 495/LOR 3A resist bilayer [7]. Imprint temperatures of at least $70^{\circ} \mathrm{C}$ above the glass transition temperature $\mathrm{Tg}=105^{\circ} \mathrm{C}$ of the PMMA allowed an optimal fabrication yield of $97 \%$. Line-widening effects related to the oxygen plasma etch resulted in beams that were 20-30 $\mathrm{nm}$ wider than the design dimensions.

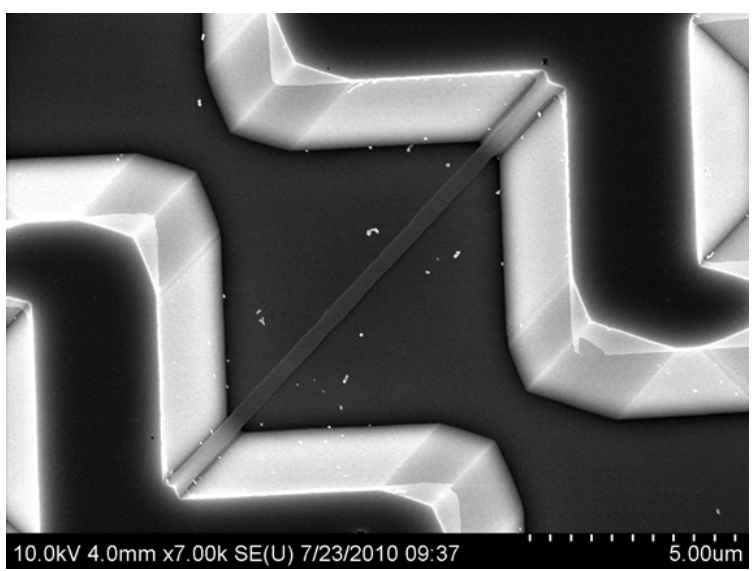

Fig. 4 Scanning electron micrograph of a $300 \mathrm{~nm}$ wide SiCN resonator element fabricated by imprint lithography

The minimum resonator width obtained using a $200 \mathrm{~nm}$-thick resist bilayer was approximately $120 \mathrm{~nm}$ (Fig. 5). The fabrication yield however rapidly dropped to below $50 \%$ for narrower structures. These $120 \mathrm{~nm}$-wide structures are however the narrowest suspended structures realized via nanoimprinting. Given the hightensile stress present in the device layer, resist imperfections such as nicks along the device edge were found to be highly detrimental to the successful realization of sub-100 $\mathrm{nm}$ wide suspended structures. Imperfection of the resist undercut profile is likely to cause the observed edge roughness. This being said, these are the narrowest suspended structures ever realized at high-yield using a nanoimprinting method. A complete resonant characterization of these imprinted devices will be presented.

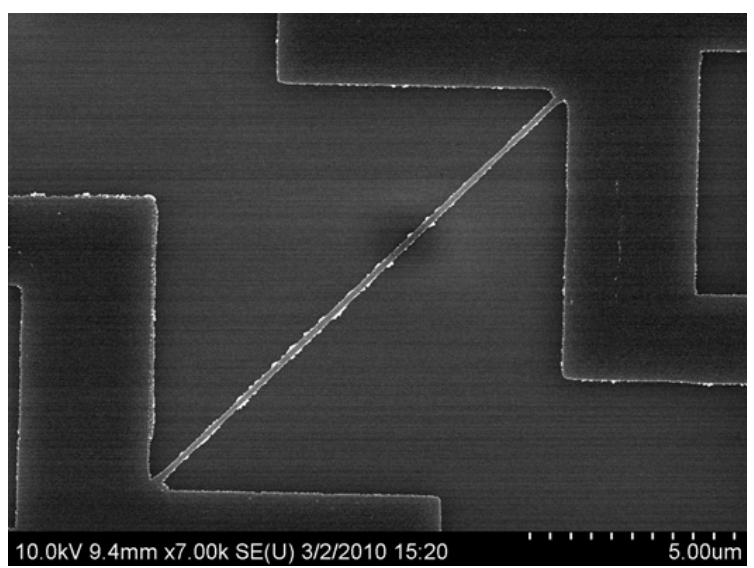

Fig. 5 Scanning electron micrograph of a $120 \mathrm{~nm}$ wide SiCN resonator fabricated by imprint lithography. 
Figure 6 now shows arrays of nanomechanical resonators produced by the bottom-up CVD method [8].

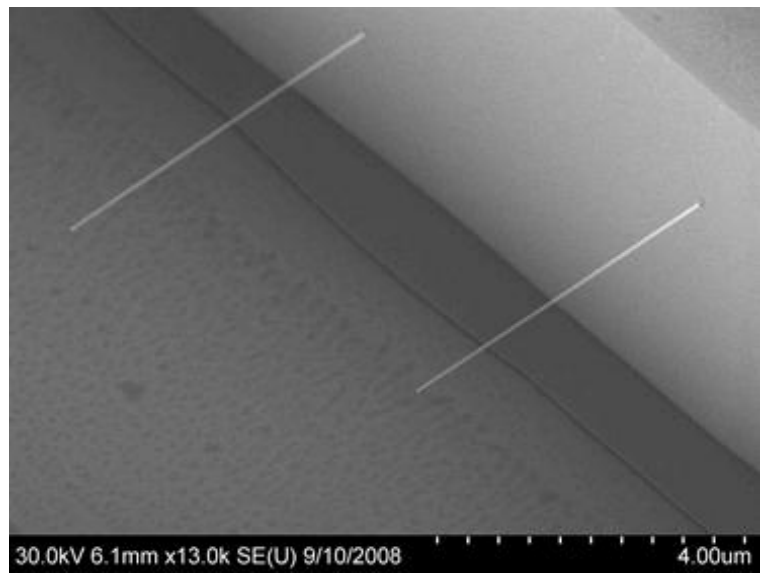

Fig. 6. 20 nm-wide bottom-up silicon nanoresonators produced by chemical vapor deposition.

In a typical experiment involving these resonant silicon nanowires, the capture of streptavidin caused a frequency shift of $120 \mathrm{kHz}$, corresponding to an attached mass of $300 \mathrm{ag}$. This added mass corresponded to a streptavidin surface density of 0.3 molecules per $100 \mathrm{~nm}^{2}$. Given that a streptavidin molecule occupies an area of $100 \mathrm{~nm}^{2}$, this experimental finding indicates that the streptavidin covers approx. $30 \%$ of the nanowire surface. Finally, exposure of similarly biotinylated nanowires to a solution of pre-saturated streptavidin showed negligible shifts, demonstrating the specificity of the capture. Finally, no significant frequency shifts were observed in the absence of the surface chemistry steps.

\section{Summary}

Large arrays of nanomechanical resonators as narrow as $8 \mathrm{mn}$ have been produced using a novel SiCN surface machining method. These arrays were used to detect antigen-antibody binding events without the need of extrinsic markers. The specific detection of protein-A using single domain antibody fragments (sdAb) was demonstrated as proof of concept.

This SiCN fabrication technology was recently employed in conjunction with nanoimprint lithography, demonstrating its amenability to cost-efficient manufacturing.
Arrays of resonant nanowires were also produced using a bottom-up CVD approach. The specific detection of streptavidin was accomplished with these devices.

\section{Acknowledgements}

The author wish to acknowledge the contributions of all graduate students and postdocs that participated in this work: Lee Fischer, Miro Belov, Csaba Guthy, Vincent Wright, Amit Singh, Mohammad Mohammad, Alexander Janzen, Somayyeh Poshtiban, and Wei Zheng.

Growth of bottom-up nanowires was performed at Hewlett-Packard Labs by Nate Quitoriano, under the supervision of Ted Kamins.

This work was supported by the National Research Council of Canada, the Province of Alberta, and the Natural Science and Engineering Research Council of Canada.

\section{References}

[1] L.M Fischer, N. Wilding, M. Gel, S. Evoy, J. of Vacuum Science \& Technology B 25, 33 (2007).

[2] D. W. Carr, S. Evoy, L. Sekaric, J. M. Parpia, and H. G. Craighead, Appl. Phys. Lett., 75, 920, (1999).

[3] Y. Cui, Q. Wei, H. Park and Ch. M. Lieber, Science 293, 1289 (2001).

[4] M. S. Islam, S. Sharma, T. I. Kamins and R. S. Williams, Nanotechnology 15, L5 (2004).

[5] C. Guthy, R. Das, B. Drobot, and S. Evoy, J. Appl. Phys. 108, 014306 (2010).

[6] M. A. Mohammad, S. K. Dew, S. Evoy, and M. Stepanova, Microelectronic Engineering 88, Issue 8, 2338-2341, (2011).

[7] A. Janzen, S. Poshtiban, A. Singh, and S. Evoy, submitted to Journal of Micro/Nanolithography, MEMS, and MOEMS.

[8] M. Belov, N. J. Quitoriano, W. K. Hiebert, S. Sharma, T. I. Kamins, and S. Evoy, J.Appl. Phys. 103, 074304 (2008). 